\title{
AUTO-EXAME DE MAMAS: IDENTIFICAÇĀO DE ALGUNS FATORES QUE INFLUENCIAM SUA PRÁTICA
}

\author{
Magda Rojas Yoshioca* \\ Doralice de Souza **
}

YOSHIOCA. M.R.: SOUZA. D. Auto exame de mamas: identificaçāo de alguns fatores que influenciam sua pratica. Rev. Eec. Enf. USP, v. 28, n.2, p. 215-26, ago. 1994.

O presente estudo teve por objetivo identificar fatores que influenciam a prática do auto-exame de mama (AEM) realizado por enfermeiras/docentes. Metodologia: foram entrevistadas $70(78,6 \%)$ das 89 profissionais que lecionam em uma instituiçäo pública da cidade de São Paulo. Resultados e conclusão: tadas reconhecem a importância da realizą̧āo do AEM. entretanto, $20,0 \%$ da populaçāo não o realiza. Os fatores identificados que influenciam a prática do AEM pelo grupo estudado foram: conhecimento técnico sobre cáncer de mama, vontade de detectar possivels alteraçóes precocemente $(80,0 \%)$, falta de habilidade para identificar alteraçōes, medo de descobrir alteraçōes e desleixo, possivelmente sob influência de suas crenças e valores.

UNITERMOS: auto-exame de mama, crenças, valores, praticas de saude.

\section{INTRODUÇÃO}

O câncer de mama tem sido responsável por milhares de mortes de mulheres no mundo.

ALMEIDA $^{1}$ mostra a estimativa americana de morbi-mortalidade segundo sexo e localizaçāo do câncer, identificando que a morbidade de câncer de mama é de 28 e a mortalidade, de 18 por 100.000 mulheres.

Segundo COELHO et $\mathrm{al}^{6}$ os dados do Ministério da Saúde revelam que 25,0\% das mortes de mulheres por câncer são causadas pelos câncer de mama e pelo de colo uterino.

No Brasil, encontram-se diferenças regionais quanto à incidência de câncer de mama. As regiões nordeste e centro-oeste detêm os maiores indices (Recife - 90,4/100.000 mulheres, Goiânia-62,3/100.000 mulheres), enquanto as regiōes sudeste e sul apresentam as menores taxas (São Paulo 41,5/100.000 mulheres, Porto Alegre - 34,6/100.000 mulheres $)^{4}$.

- Prof. da Faculdade de Enfermagem e Nutriçăo da UFMT Mestre em enfermagem

- Prof. da Escola de Enfermagem da USP. Doutor em Saúde Pública. 
As diferenças regionais quanto as taxas de óbito também são apontadas por ALMEIDA ${ }^{1}$ ao informar que $17,4 \%$ dos óbitos femininos por neoplasias malignas do sudeste constituem a mais alta taxa.

Outra informação importante é que o câncer de mama incide mais em mulheres com mais de 35 anos de idade, e que, após os 60 anos é a primeira hipótese de câncer a ser considerada ${ }^{6,10}$.

Entretanto, sabe-se que o exame periódico de colo uterino e de mamas poderiam evitar, em grande parte, óbitos por neoplasias malignas na mulher ${ }^{5}$.

Quanto ao hábito das pessoas auto-examinarem partes de seus corpos, MAMEDE $^{12}$ encontrou, em sua pesquisa, que $38,9 \%$ das mães e $86,8 \%$ das alunas entrevistadas se auto-examinavam. Encontrou também que apenas $17,0 \%$ da amostra estudada procuraram serviços de saúde com o objetivo apenas de supervisão de saúde.

Sabe-se que, ao planejar um programa educativo para a saúde, o profissional deve levar em consideração o conhecimento e as crenças que a clientela a ser atingida tem sobre o tema em questão. Mas isso não é tão simples quanto parece à primeira vista.

O modelo de crenças em saúde proposto por ROSENSTOCK ${ }^{17}$ considera os aspectos sócio-demográfico-psicológicos e estruturais do comportamento humano para ajudar nas percepçōes dos clientes acerca de sua suscetibilidade pessoal à doença em foco; na sua percepçāo acerca do poder que tal agravo tem de provocar sérias consequências à sua saúde (severidade) e, finalmente. na percepção de quais beneficios (redução da suscetibilidade e da severidade) 0 cliente terá ao praticar uma ação particular enquanto comportamento preventivo. Considera que existem, ainda, barreiras para a percepçāo dos benefícios. como custo ou dor, por exemplo.

As crenças sobre a saúde de uma pessoa são consequência do que ela pensa e acredita sobre saúde e doença bem como de suas atitudes em relação às mesmas ${ }^{16}$.

Acredita-se que, se não houver sensibilização e envolvimento da clientela e reconhecimento, pelos profissionais de saude sobre os fatores relativos às crenças e valores da clientela a ser atendida, pouco se poderá influenciar nos aspectos comportamentais preventivos.

Um dos chamados métodos preventivos é o auto-exame que detecta precocemente alteraçāo da mama ${ }^{10}$. Cerca de $90,0 \%$ dos cânceres de mama são descobertos pela própria mulher, não necessitando do médico para esta descoberta, seja ela casual ou devido à exame periódico das mamas realizado por ela mesma ${ }^{10.11}$.

Acredita-se que os profissionais de saúde, entre eles as enfermeiras/docentes devam estar atentas para a importancia do auto-exame de mama e realizá-lo periodicamente, pois têm ao seu alcance informaçōes sobre como obter maior conhecimento sobre seu corpo.

Acredita-se ainda que, os cursos para formaçāo de profissionais da área da saúde, estejam alertando os futuros profissionais sobre a importância das medidas preventivas e de promoção à saúde, conforme demonstram os dados de BERGAMASCO; TSUNECHIRO ${ }^{3}$ sobre fontes de informaçāo acerca do 
auto-exame de mama. Espera-se portanto que, à partir dos conhecimentos específicos sobre câncer de mama e formas de detecção precoce, as profissionais passem a adotá-las em suas experiências pessoais e profissionais.

\section{OBJETIVO}

Diante do exposto, o objetivo do presente estudo é:

- Identificar fatores que influenciam a prática do auto-exame de mama por enfermeiras/docentes.

\section{METODOLOGIA}

A presente pesquisa é um estudo exploratório descritivo sobre a prática de realização de auto-exame de mama entre mulheres que possuem formaçào relativa à promoção e cuidados à saúde e exercem atividades de docència nesta área.

Do total de 89 enfermeiras/docentes que trabalham em uma instituição pública da cidade de São Paulo, participaram deste estudo $70(78,6 \%)$ profissionais.

$O$ instrumento contém itens referentes à caracterização da população estudada (parte $A$ ) e dados relativos à prática ou não do auto-exame de mamas (parte B) (ANEXO I). O estudo piloto do instrumento foi realizado com 5 enfermeiras/docentes, da mesma instituição. Constatando-se que nào houve necessidade de alteraçōes do instrumento, essas profissionais passaram a fazer parte da população alvo da pesquisa.

A coleta de dados foi realizada no mês de novembro de 1992 . Uma das pesquisadoras apresentava-se à profissional explicando o objetivo da pesquisa, solicitava sua participação e assegurava-lhe o seu anonimato. Após aceitação, passava a entrevistá-la.

Os dados foram agrupados e são apresentados sob forma de tabelas e quadro. Algumas vezes foram transcritas as respostas na integra.

\section{RESULTADOS E COMENTÁRIOS}

Em relação à caracterização da população estudada encontrou-se que, das mulheres entrevistadas, $77,2 \%$ têm idade acima de 35 anos.

Curiosamente a maior parte da populaçāo do estudo encontra-se na faixa etária que favorece maior risco de apresentar neoplasia mamaria. Confirmam esta afirmação os achados do ALMEIDA ${ }^{1}$, COELHO et al $^{6}$ e da FUNDAÇĀO OSWALDO CRUZ?.

Em relação ao risco de desenvolver câncer de mama e a idade da mulher, Silva, citado por ALMEIDA ${ }^{1}$, refere que há divergència entre os pesquisadores. Alguns deles consideram que o cancer de mama tem pior prognostico 
quando a mulher tem mais de 40 anos e, quanto mais idosa ela for, menor sua sobrevida. Outros, no entanto, afirmam que a mulher com até 35 anos apresenta pior prognóstico.

Apesar das divergências, os dados oficiais sobre mortalidade, revelam que o número de óbitos por neoplasia maligna da mama é maior em mulher acima de 30 anos ${ }^{4,7}$.

Quanto aos dados relativos ao tempo em que as entrevistadas estāo formadas e exercem atividades de ensino verifica-se que a grande maioria da populaçāo $(98,6 \%)$ tem mais de cinco anos de formada em enfermagem. Este fato pode fazer supor que as entrevistadas tiveram muito mais oportunidades de acesso à informação sobre a importancia do auto-exame de mama do que a população leiga ou, ainda, se fossem simples acadêmicas ou recém-formadas, por força mesmo do meio profissional em que vivem.

Outra coisa importante seria buscar informações sobre quando este assunto passou a ser introduzido ou mais discutido nos cursos de graduação em enfermagem. Entretanto, essa informação não estava disponivel na literatura consultada.

Geralmente os profissionais que exercem a docência estão ligados aos grandes centros de pesquisas, ou com eles estabelecem contato ou deles podem obter informaçōes. Isto só vem reforçar que a população objeto de estudo teria maior facilidade de acesso à obtenção de conhecimentos sobre o assunto em pauta.

Quanto à titulação das entrevistadas $52,9 \%$ possuem título de mestre, $22,9 \%$ de doutor e $11,4 \%$ possuem apenas a graduação.

Quanto à área de atuaçāo das enfermeiras docentes entrevistadas encontrou-se que $25,7 \%$ desenvolvem atividades nas áreas que atendem a mulher ("Obstétrica/Neonatal" e "Saúde Coletiva/Preventiva"), 18,6\% em Médico-cirúrgica, 14,3\% em Administração Aplicada à Enfermagem, 8,6\% em Centro Cirúrgico e Central de Material Esterilizado, Fundamentos/Ética e Psiquiátrica, $7,1 \%$ em Doença Infecto Parasitária e Pediátrica e $1,4 \%$ em Informática. tadas.

A prática do auto-exame de mama é realizado por $80,0 \%$ das entrevis-

Embora o percentual de enfermeiras docentes que executam o auto-exame de mama seja alto $(80,0 \%)$ em relação aos estudos citados anteriormente, ele ainda é inferior aos dados encontrados por BERGAMASCO; TSUNECHI$\mathrm{TO}^{3}$, ainda que nessa pesquisa, a população estudada o faça com periodicidade variável.

Outros estudos têm mostrado que a frequêencia da prática do auto exame de mama varia.

MAMEDE; PELÁ ${ }^{13}$ entrevistando mães e alunas que cursavam o segundo grau, identificou que $76.3 \%$ das mulheres entrevistadas faziam o auto-exame, sendo maior a incidência entre as adolescentes. As autoras mostram a importância de reforçar o comportamento dessas adolescentes para que essa prática seja incorporada a seus estilos gerais de vida. 
No trabalho de $\mathrm{AMADOR}^{2}$ com mulheres que frequentavam ambulat6rios de ginecologia e clínica médica foi identificado que apenas $24,75 \%$ das mulheres realizavam auto-exame de mama.

Os estudos de GRAY ${ }^{8}$ com mulheres americanas de zona rural demonstram que $43,0 \%$ das mulheres realizam o auto-exame da mama.

STILLMAN ${ }^{18}$ encontrou que a prática do auto-exame de mama foi de $72,8 \%$ das mulheres que pertencem a uma determinada organização feminina por ele estudada.

HEYMAN et $\mathrm{al}^{9}$ referem que em 1989, a Sociedade Americana do Câncer estimou que em 143.000 mulheres poderia ser diagnosticado câncer de mama e que houve 43.000 óbitos por essa etiologia. Os autores afirmam que, a despeito dessa estatística, somente $24,0 \%$ das mulheres americanas dizem que praticam mensalmente o AEM. Eles atribuem esse fato a falta de conhecimento, medo de encontrar algo, sentimentos de culpa ou embaraço. Ainda os mesmos autores constataram, em sua pesquisa com enfermeiras de clínica médico-cirúrgica que, apesar de $99,9 \%$ delas estarem aptas para ensinar o auto-exame de mama apenas $26,0 \%$ delas o fazem mensalmente.

Em virtude das características da populaçăo do presente estudo considera-se que o percentual encontrado, das entrevistadas que não realizam o auto-exame de mama é alto $(20,0 \%)$, uma vez que são mulheres. especialistas nas áreas de Enfermagem Obstétrica/neonatal e de Saúde Coletiva/Preventiva e lidam com clientes e alunas de enfermagem do sexo feminino.

No entanto, sabe-se que, mudança de comportamento năo depende apenas do conhecimento. Goldsen, citado por MAMEDE ${ }^{12}$ lembra que "o processo chave para mudança de comportamento nảo está simplesmente na cognição, mas a cognição tem que estar inserida no back-ground de socialização dos indivíduos. O processo cognitivo ocorre como um todo, onde se estabelecem associações congruentes entre hábito, comportamento e valores".

MAMEDE $^{12}$ acredita que tendo a pessoa conhecimento adequado e suficiente sobre o câncer, ela teria condiçōes de tomar decisões sobre a realização de ações preventivas. Entretanto, a propria autora e outros pesquisadores como HEYMAN et $\mathrm{al}^{9}$ chamam atenção sobre o fato de que 0 conhecimento "não leva, por si só, à mudança de comportamento".

No presente estudo, a realização do auto-exame de mama é maior entre as entrevistadas com idade entre 40 e 45 anos e acima de 5 anos de docência, semelhantes aos achados de BERGAMASCO; TSUNECHIRO ${ }^{3}$, GRAY ${ }^{8}$ e NEMCEK ${ }^{15}$

Dentre as entrevistadas, as que estão mais em contato com a clientela feminina ("Obstétrica/Neonatal" e "Saúde Coletiva/Preventiva") são as que mais praticam o auto-exame. Corroboram estes dados os achados de BERGAMASCO; TSUNECHIRO ${ }^{3}$.

Todas as entrevistadas acham importante realizar o auto-exame de mama, coincidindo com a opinião das enfermeiras de hospitais gerais que os autores acima estudaram ${ }^{3}$.

As falas das entrevistadas podem melhor elucidar a importância que dão à realização do auto-exame de mamas: “é um momento de contato com 
o próprio corpo", “é fácil, simples, rápido e de custo zero", "fácil. nào precisam ir até o médico...", "para conhecimento do próprio corpo e sua valorização...", "porque é comprovadamente eficiente".

Algins achados chamam a atenção como o significado que algumas entrevistadas (07) dão ao contato com o próprio corpo, para se conhecerem melhor como mulher; outras 4 consideram o procedimento de fácil execução.

Todas as entrevistadas acham importante a realização do auto-exame de mama, contudo $20,0 \%$ delas não o praticam.

Os motivos mencionados para a realização do AEM encontram-se na Tabela 1.

Dentre os motivos que as entrevistadas atribuem ao exame, mesmo entre aquelas que não o realizam, destacaram-se em ordem decrescente: "Detecção de problema de mama" (35,8\%), "Prevenção de Câncer de mama" $(22,9 \%)$ e "Detecção de câncer de mama" $(17,1 \%)$.

As falas de algumas entrevistadas mostram o grau de conhecimento técnico e o enfoque que elas acreditam que deve ser dado à saúde: "Segundo estatística, cerca de $90,0 \%$ dos casos de alterações da mama são detectadas pela pessoa e não pelo médico", "Atualmente temos que trabalhar na prevenção e diagnóstico precoce... não só em oncologia”.

TABELA 1: MOTIVOS PELOS QUAIS AS ENFERMEIRASDOCENTES, ACHAM IMPORTANTE FAZER AUTO-EXAME DE MAMA. SĀO PAULO, 1992.

\begin{tabular}{|c|c|c|c|c|c|c|}
\hline \multirow[t]{3}{*}{ MOTIVO } & \multicolumn{6}{|c|}{ REALIZAÇÄO DE AUTO-EXAME DE MAMA } \\
\hline & \multicolumn{2}{|c|}{$\operatorname{SIM}$} & \multicolumn{2}{|c|}{ NẢO } & \multicolumn{2}{|c|}{ TOTAL } \\
\hline & f & $\%$ & $\mathrm{f}$ & 8 & $f$ & $\%$ \\
\hline Detecção de probiema de mama & 19 & $27,2^{*}$ & 6 & 8,6 & 25 & 35,8 \\
\hline Prevenção de Câncer de mama & 13 & 18,6 & 3 & 4,3 & 16 & 22.9 \\
\hline Detecçāo de Câncer de mama & 12 & 17,1 & $\cdot$ & & 12 & 17.1 \\
\hline Prevençāo de problema de mama & 3 & 4,3 & 2 & 2.8 & 5 & 7,1 \\
\hline Forma de prevençāo/detecçāo & 6 & 8,6 & 3 & 4,3 & 9 & 12,9 \\
\hline Outro & 3 & 4,2 & $\cdot$ & & 3 & 4.2 \\
\hline TOTAL & 56 & 80.0 & 14 & 20.0 & 70 & 100.0 \\
\hline
\end{tabular}

- Feito aproxımaçáo para efeito de caiculo

MAMEDE $^{12}$ questiona sobre o significado do AEM e apresenta claramente sua própria resposta: "consiste, na busca sistemática de alteraçōes que levem ao diagnóstico precoce de câncer". Em poucas palavras: procura do câncer. Talvez isto possa explicar parcialmente o fato de que $20,0 \%$ da população não realiza o auto-exame de mama, como será visto nos comentários do Quadro 1.

LAGANÁ et al ${ }^{10}$ citam como fatores predisponentes à realizaçāo do AEM a valorização do procedimento; conhecimento sobre câncer de mama; expe- 
riência pessoal, com amigas e familiares; crenças e valores sobre o câncer e o cuidado com a saúde, entre outros.

$E$ interessante notar que a maioria $(60,0 \%)$ das entrevistadas evitaram mencionar a palavra câncer. Este fato pode ser melhor compreendido pelo estudo sobre mitos e preconceitos relativos ao câncer que VALBOX ${ }^{19}$ apresentou no Royal Society of Health nos Estados Unidos, em 1984. Neste estudo o autor afirma que "câncer é uma palavra que provoca uma reação emocional imediata, uma resposta que parece não ter relação com o pensamento racional, com o conhecimento profundo ou com as regras individuais da sociedade. A mesma reação parece ocorrer com médicos, enfermeiras, professores, universitários, pesquisadores políticos e trabalhadores sem capacitação técnica. Isso também ocorre em pacientes, familiares, amigos ou aqueles que têm câncer, em pessoas que pensam que câncer pode ocorrer somente para outras pessoas. Em todos esses grupos, os sentimentos e fantasias surgem apenas na mençãa da palavra câncer. e parecem ser praticamente idênticas".

Outro fato a ser ressaltado é o emprego inadequado da terminologia, quando 42,9\% das entrevistadas referem-se ao AEM como "Prevenção de Câncer de mama", "Prevenção de problema de mama" e "Forma de prevenção...", pois sabe-se que o AEM consiste na busca de alteraçōes da mama que levem à deteç̧ão precoce do câncer, não impedindo que processos neoplásicos surjam e sim que melhore seu prognóstico.

Os principais motivos que levam as entrevistadas a não realizarem o AEM são, em ordem decrescente, segundo seus próprios depoimentos ( $Q$ uadro 1): sabe fazer mas tem dificuldades de identificar alteraçooes, medo de achar alguma alteraçào e desleixo.

QUADRO 1: MOTTVOS PELOS QUAIS 14 ENFERMEIRASIDOCENTES NÃO FAZEM AUTO-EXAME DE MAMA SÄO PAULO, 1992.

\begin{tabular}{lr}
\hline MOTIVO* & $\mathrm{f}$ \\
\hline Sabe fazer mas tem dificuldades de identificar alteraçöes & 6 \\
Medo de achar alguma alteraçào & 4 \\
Desleixo & 4 \\
Esquecimento & 2 \\
Marido é medico e faz & 2 \\
Tem outras prioridades & 1 \\
Faz controle com ginecologista & 1 \\
Não sabe fazer auto-exame & 1 \\
\hline TOTAL & 21 \\
\hline
\end{tabular}

- As entrevistadae citaram um ou mals motivos

No estudo de LAGANÁ et al ${ }^{10}$ sobre a identificaçāo dos conhecimentos, atitudes, habilidades e práticas relativos ao AEM visando a elaboraçāo de programas educativos, as respostas dadas pela população estudada foram agrupadas em fatores comportamentais relacionados à mulher: falta de 
habilidades e dificuldades para palpar a mama, medo de encontrar alterações e esquecimento, dentre outros.

Falta de habilidade ou dificuldade para identificaçāo de alteraçōes também foram encontradas no estudo de HEYMAN et al $^{9}$.

O medo de achar alguma alteração aparece, também, nos trabalhos de HEYMAN et $\mathrm{al}^{9}$ e STILLMAN ${ }^{18}$.

A fala na íntegra de algumas entrevistadas, melhor exemplificam esses motivos: "Medo. Tenho problemas de ordem psicológica. Não tenho coragem de fazer... deixo para o médico, morro de medo de encontrar alguma alteração... e pensar que sou enfermeira", "Tenho medo de ter alguma coisa... tenho antecedentes familiares", "Falta de preocupação porque o marido faz", "Não lembro de fazê-lo", "Tenho displasia, faço controle periódico, mamografia e ginecologista".

Os dados sobre o momento da realização do auto-exame de mama, em relação ao ciclo menstrual, encontram-se agrupados na Tabela 2.

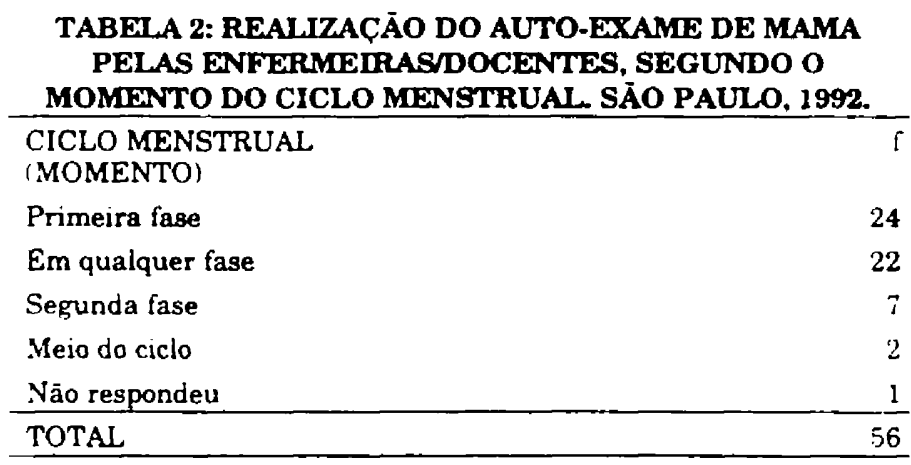

O momento mais citado é a primeira fase $(42,8 \%)$ do ciclo menstrual. que é considerado como mais adequado, devido ao fato das mamas encontrarem-se mais flácidas e menos doloridas, como sugerem COELHO et $\mathrm{al}^{6} \mathrm{e}$ MARTIN; BORRAS ${ }^{14}$. No entanto agrupando-se em qualquer fase e na segunda fase do ciclo menstrual, obtem-se $51,8 \%$ de mulheres que realizam seu auto-exame nestes momentos. Contudo, estas podem apresentar desconforto por estarem as mamas túrgidas quando está próximo o início da menstruação ou seja no fim do ciclo.

Os dados referentes a periodicidade da realização do AEM encontram-se na Tabela 3 na página seguinte.

No presente trabalho encontrou-se que $62.5 \%$ das entrevistadas realizam o AEM pelo menos uma vez no mês, enquanto no estudo de BERGAMASCO; TSUNECHIRO ${ }^{3}$ com enfermeiras hospitalares o percentual é menor $(40,0 \%)$.

A prática do auto-exame de mamas é maior entre as entrevistadas com mais de 50 anos, coincidindo com os achados de BERGAMASCO; TSUNECHI$\mathrm{RO}^{3}$ e GRAY ${ }^{8}$. 
TABELA 3: PERIODICIDADE DA REALIZAÇĀO DO AUTO-EXXME DE MAMA PELAS

ENFERMEIRASDOCENTES. SĀO PAULO, 1992.

\begin{tabular}{lrr}
\hline PERIODICIDADE & f & $\%$ \\
\hline Mensal & 29 & 51,8 \\
Esporádico & 10 & 17,9 \\
Várias vezes no mès & 6 & 10,7 \\
De 2 a 3 meses & 4 & 7,1 \\
Bimensal & 2 & 3,6 \\
Outra & 4 & 7,1 \\
Nảo respondeu & 1 & 1,8 \\
\hline TOTAL & 56 & 100,0 \\
\hline
\end{tabular}

As falas de algumas entrevistadas revelam fatores relacionados com a prática e periodicidade do auto-exame de mama, como as experiências pessoais e familiares: "Semanalmente, às vezes periodo maior... Não me preocupava antes porque nunca tive alteração na mama e nem problemas oncológicos na familia... não me preocupei até 8 anos atrás até ser histerectomizada".

$O$ fato de que algumas $(10,7 \%)$ das entrevistadas fazem o AEM mais de uma vez por mès, pode fazer supor que estão atentas e/ou preocupadas com a deteç̧ão precoce do câncer de mama, talvez pelas experiências que experimentaram em sua vida profissional e/ou pessoal.

Outro achado interessante é a diferença encontrada entre o número de profissionais que dizem realizar o auto-exame $(80,0 \%)$, com aqueles referentes ao exame sistemático das mamas feito mensalmente $(62,5 \%)$. Esta diferença sugere um aumento na possibilidade das entrevistadas terem mais dificuldades em identificar alterações, bem como esquecerem de realizar tal exame.

A maioria $(77,8 \%)$ das entrevistadas realiza o auto-exame de mamas há mais de 5 anos. Há diferença entre estes dados, o tempo em que estão formadas e o tempo em que desenvolvem atividade de docência. Confirmando que nāo basta apenas ter conhecimento para incorporar uma prática, parece que outros fatores também interferem como as crenças e os valores e experiências individuais, como foi dito anteriormente. Isto pode ser parcialmente percebido através das falas das entrevistadas: "Após histerectomia há 7 anos", "Há 5 ou 6 anos, após saber que colega estava com Ca", "Desde que entrei no climatério... vou ao médico de 6/6 meses", "Desde que aprendi no curso de graduação", "Desde que me formei enfermeira obstétrica", "Por causa de um programa que vi na TV".

\section{CONCLUSĀO}

No presente estudo verificou-se que $80,0 \%$ das entrevistadas realizam o auto-exame de mamas (AEM), embora nem sempre com a periodicidade e época preconizadas. 
Apesar de que todas $(70=100,0 \%)$ acham importante a realizaçāo do AEM, 20,0\% delas não o praticam.

Constatou-se que, semelhante aos achados da literatura consultada, os fatores que influenciam a prática do AEM, na população estudada são: conhecimento técnico sobre câncer de mama, vontade de detectar possíveis alteraçōes precocemente $(80,0 \%)$, falta de habilidade em identificar aiterações, medo de achar alguma alteração e desleixo, possivelmente sob influència de suas crenças e valores sobre saúde e, ainda, à partir de experiência pessoal com parentes, amigos e clientes que tiveram câncer.

YOSHIOCA, M.R.; SOUZA. D. Breast self examination: identification of factors that influency the practsce. Rev. Esc. Enf. USP, v. 28, a.2, p. 215-26, aug. 1994.

The aim of this study was to identify the factors that influences the practice of breast self-examination (BSE) by nursing teachers. Seventy profissionals were interwied. Although all of them judged important the realization of the BSE, 20\% didn't practice it. The identified factors were: the techinical knowledge concerning breast cancer, the desire of early detection of possible alterations (80\%), the lack of ability to identify those alterations, the fear of discovering some alterations and also negligence, possibly influenced by their beliefs and values about health.

UNITERMS: breast self examination, beliefs, values, health practice.

\section{REFERÊNCIAS BIBLIOGRÁFICAS}

1. ALMEIDA, A.M. Câncer de mama: análise de fatores de risco sob a perspectiva da teoria de Kurt Lewuin. Rio de Janeiro, 1991.61p. Dissertaçāo (Mestrado) -Escola de Enfermagem Ana Neri, Universidade Federal do Rio de Janeiro.

2. AMADOR, M.V.P. Contribuiçāo ao estudo do auto-exame da mama como método de detecção precoce do câncer. São Paulo, 1975. 83p. Dissertaçáo (Mestrado) -Escola de Enfermagem, Universidade de Sảo Paulo.

3. BERGAMASCO, R.B.B.; TSUNECHIRO, M.A. A enfermeira na prática e no ensino do auto-exame da mama. Ci e Cult., v.40, n.7, p.63. Suplemento/Apresentado à 44 Reunaão Anual da SBPC, São Paulo, 1992 - Resumal

4. BRASIL. Ministério da Saúde. Instituto Nacional de Cáncer (INCa). Câncer no Brasil: dados dos registros de base populacional. Rio de Janeiro, INCa, 1991.

5. BRASIL. Ministério da Saúde. Assistência integral à saúde da mulher: base de açào programatica. Brasilia, Centro de Documentação do Ministério da Saude, 1984.

6. COELHO, A. et al. Câncer de mama. In: FUNDAÇĀO ONCOCENTRO DE SĀO PALLO. Câncer de colo uterino, mama e pele. São Paulo, 1991 p.17-28.

7. FUNDAÇẢO OSWALDO CRUZ (FIOCRUZ). Câncer : mortalidade no Brasil. Rio de Janeiro, FIOCRUZ, 1991. (Dados, n.15)

8. GRAY, M.E. Factors related to practice of breast self-examination in rural women. Cancer Nurs., v.13, n.2, p.100-7, 1990. 
9. HEYMAN, E. et al. Is the hospital setting that place for teaching breast self-examination? Cancer Nurs., v.14, n.1, p. 35-40, 1991.

10.LAGANÁ. M.T.C. et al. Auto exame de mamas: identificação dos conhecimentos, atitudes, habilidades e praticas (CAMP) requeridos para elaboração de propostas educativas. Rev.Esc.Finf.USP. , v.24, n.2, p.281-99, 1990.

11.LUDWICK. R. Breast examination in the older adult. Cancer Nura, v.11, n.2, p.99.102, 1988.

12.MAMEDE. M.V. O câncer no conterto dos cuidados primários com a saúde. Ribeirão Preto, 1985. 177p. Tese(Doutorado)- Escola de Enfermagem de Ribeiráo Preto. Universıdade de Sāo Paulo

13.MAMEDE, M.V.; PELA, N.T.R. Fatores associados à realizaçào do auto-exame das mamas. Femina, v.16, n.6, p.486-92, 1988.

14.MARTIN, C.H.; BORRAS. C.M. La exploración de mama: primer eslabón en la prevención del cancer. Rev.Infirm. v. 10, a.107/108, p.23-9. 1987.

15.NEMCEK. M.A. Factors influing black women's breast self examination practice. Cancer Nurs., v. 12, v.6, p.339-43, 1989.

16.PEDROSA, L.A.K Crenças das pessoas portadoras de hanseniase sobre sua doença: base para a compreensáo de suas açōes em saude. Ribeirảo Preto, 1991. 157p. Dissertaçào (Mestrado) -Escola de Enfermagem de Ribeirão Preto. Universidade de São Paulo.

17.ROSENSTOCK. I.M. The health belief model and preventive health behavior. Health Educ.Monogr., v.2, n.4, p.354-86, 1974.

18.STILLMAN, M.J. Women s health beliefs about brest cancer and breast self-examination. NursRes, v.26, n.2, p. 121-7, 1977

19.VALBOX, M.S.C. Cancer: myths and misconceptions. J.R.Soc.Health . v.104, n. 5, p. 161-6, 1984. 


\section{ANEXO I}

VOCÊ ENFERMEIRADOCENTE GOSTARIA DE CONTRIBUIR PARA A IDENTIFICAÇĀO DE FATORES QUE INFLUENCIAM NA PRÁTICA DO AUTO EXAME DE MAMA? ENTÃO RESPONDA AS QUESTŌES ABAIXO. ESTAS INFORMAÇÕES PODEM SERVIR PARA REORIENTAR PROGRAMAS DE PREVENÇĀO DE CÁNCER DE MAMA.

A - DADOS GERAIS DE IDENTIFICAÇĀO

1 - IDADE (EM ANOS): 2 - FORMATURA HÁ ANOS

3 - TEMPO DE DOCENCIA (EM ANOS): 4 - TITULAÇÃO:

5 - ÁREA DE ATUAÇẢO:

B - DADOS RELATIVOS AO EXAME DE MAMA

6 - VOCE ACHA IMPORTANTE O AUTO EXAME DE MAMA?

SIM( ) NĀO( )

POR QUE?

7 - VOCÉ FAZ AUTO EXAME DE MAMA?

SIM( ) NẢO( )

EM CASO DE RESPOSTA AFIRMATIVA PULE PARA A QUESTẢO 09, SE NEGATIVO RESPONDA A PRÓXIMA QUESTĀO

8 - VOCE NÄO FAZ AUTO EXAME DE MAMA POR QUE:

NÄO SABE FAZER( )

SABE FAZER MAS TEM DIFICULDADE DE IDENTIFICAR ALTERAÇÕES DURANTE O EXAME ( )

. NĀO TEM TEMPO ( )

TEM OUTRAS PRIORIDADES（）

TEM OUTRO MOTIVO

(QUAL? )( )

SE A QUESTẢO 07 FOR AFIRMATIVA, RESPONDA AS QUESTÕES ABAI$\mathrm{XO}$

9 - EM QUAL MOMENTO DO CICLO MENSTRUAL VOCE REALIZA O AUTO-EXAME DE MAMA?

PRIMEIRA FASE ( )

SEGUNDA FASE ( )

EM QUALQUER FASE ( )

10 - PERIODICIDADE DA REALIZAÇÃO DO AUTO-EXAME DE MAMA:

11 - HA QUANTO TEMPO VOCÊ REALIZA O AUTO-EXAME DE MAMA? OBRIGADA 